\title{
Cervical mucus monitoring prevalence and associated fecundability in women trying to conceive
}

\author{
Emily Evans-Hoeker, M.D. ${ }^{a}$, David A. Pritchard, M.S. ${ }^{b}$, D. Leann Long, M.S. ${ }^{b}$, Amy H. \\ Herring, Sc.D. ${ }^{b, c}$, Joseph B. Stanford, M.D., M.S.P.H. ${ }^{a, c, d}$, and Anne Z. Steiner, M.D., M.P.H. ${ }^{a}$ \\ aDepartment of Obstetrics and Gynecology, University of North Carolina, Chapel Hill, North \\ Carolina \\ bDepartment of Biostatistics, University of North Carolina, Chapel Hill, North Carolina \\ 'Carolina Population Center, University of North Carolina, Chapel Hill, North Carolina \\ ${ }^{\mathrm{d}}$ Department of Family and Preventive Medicine, University of Utah, Salt Lake City, Utah
}

\begin{abstract}
Objective-To assess the use of cervical mucus monitoring (CMM) in women trying to conceive and determine whether monitoring is associated with increased cycle-specific probability of conception (fecundability).

Design-Time-to-pregnancy cohort study.

Setting-Population-based cohort.

Patient(s)-Three hundred thirty-one women trying to conceive, ages 30 to 44 years, without known infertility

Intervention(s)-None.

Main Outcome Measure(s)—CMM prevalence and fecundability.

Result(s)-During the first cycle of the study, CMM was performed consistently (checked on $>66 \%$ of pertinent cycle days) by 20 women (6\%), inconsistently (34\% to $66 \%$ of days) by 60 women ( $18 \%$ ), infrequently ( $\leq 33 \%$ of days) by 73 women $(22 \%)$, and not performed by 178 women (54\%). Cycles in which CMM was consistently performed were statistically significantly more likely to result in conception after adjusting for age, race, previous pregnancy, body mass index, intercourse frequency, and urinary luteinizing hormone (LH) monitoring. Fecundability also increased with increasing consistency of CMM.
\end{abstract}

Conclusion(s)-Among women trying to conceive, CMM is uncommon, but our study suggests that CMM-a free, self-directed method to determine the fertile window-is associated with increased fecundability independent of intercourse frequency or use of urinary LH monitoring.

C2013 American Society for Reproductive Medicine, Published by Elsevier Inc.

Reprint requests: Emily Evans-Hoeker, M.D., University of North Carolina at Chapel Hill, CB \#7570, Chapel Hill, North Carolina 27514, evanshoe@med.unc.edu.

E.E.-H. has nothing to disclose. D.A.P. has nothing to disclose. D.L.L. has nothing to disclose. A.H.H. has nothing to disclose. J.B.S. has been a consultant with Swiss Precision Diagnostics and reports grants from Women's Reproductive Health Foundation and National Institute of Child Health and Human Development. A.Z.S. has been a consultant with Roche Diagnostics and reports grants and payment for lectures from the National Institute of Child Health and Human Development. 


\section{Keywords}

Cervical mucus monitoring; fertile window; conception; fecundability

Timed intercourse during the 6-day window before and including the day of ovulation (the fertile window) is significantly more likely to result in conception $(1,2)$. In the United States, it has been estimated that over 7 million women have sought professional fertility treatment, and almost 450,000 of those women sought medical care for advice on topics such as detecting the fertile window and optimizing intercourse timing $(3,4)$. Because the duration of the luteal phase is relatively stable, the days of the fertile window can be estimated based on historical cycle length (5). In addition, a variety of self-administered methods based on symptoms or biomarkers have been developed to assist in the prospective detection of ovulation and the fertile window (4).

Urinary luteinizing hormone (LH) monitoring is one such method that is commonly used to detect ovulation; however, this method can be expensive. Urinary LH monitoring alone results in false-positive results in approximately $7 \%$ of cycles in an infertile population (6), and it does not allow for prospective determination of the entire fertile window (7). Basal body temperature (BBT) monitoring is another frequently used method; however, the temperature change may be difficult to define, and the fertile window can only be defined retrospectively (8-10).

Cervical mucus monitoring (CMM), a prospective method to detect the fertile window, is performed via vulvar observations (excluding days of menstrual flow). Internal checking of the vagina or cervix is not required. The cervical mucus $(\mathrm{CM})$ is easily classified based upon appearance and sensation. Types 1 and $2 \mathrm{CM}$ are typically present at the beginning of the menstrual cycle and are associated with dry (type 1) or damp (type 2) sensations at the vulva. Type 3 is typically characterized by thick, creamy, and whitish or yellowish CM and a damp sensation at the vulva. Type 4 is characterized by transparent and stretchy or elastic $\mathrm{CM}$ (reminiscent of raw egg whites) and a wet or slippery sensation at the vulva (11). Several studies have demonstrated that CMM is an excellent method for predicting conception probabilities. Intercourse on a day with type $4 \mathrm{CM}$ results in the highest probability of conception and type 1 the lowest (11-15). An act of sexual intercourse occurring on a day with type $4 \mathrm{CM}$ is at least two to three times more likely to result in conception than intercourse on a day with types 1 or 2 (14).

Previous trials of CMM have included at least one face-to-face training session regarding monitoring. These studies have examined the predictive value of a given type of $\mathrm{CM}$, but none have determined whether using CMM improves the timing of intercourse or shortens the time to pregnancy. Furthermore, CMM prevalence has not been assessed in an untrained, noninfertile population. Therefore, our study assessed the current prevalence and consistency of CMM in a population of women trying to conceive and determined whether CMM, without formal training, is associated with increased cycle-specific probability of conception (fecundability).

\section{Materials and Methods}

Time to Conceive (TTC) is anongoing time-to-pregnancy study approved by the institutional review board of the University of North Carolina (16). The cohort from which our subjects were obtained included English-speaking women between 30 and 44 years of age who had been trying to conceive for 3 months or less. The exclusion criteria included history of infertility, polycystic ovary syndrome, pelvic inflammatory disease, endometriosis, prior 
pelvic radiation, or a history of infertility in the partner. After consent was obtained, eligible women completed a self-reported, online baseline survey of demographics, height, weight, and medical history—for both herself and her male partner-as well as behaviors including tobacco, alcohol, and caffeine use. Women were also instructed on the use of an online daily diary to record information on vaginal bleeding, intercourse, methods and results of testing for the fertile window (if performed), as well as pregnancy test results.

Women who reported that they checked their $\mathrm{CM}$ on any given day were asked to choose the type observed that day (according to the types 1 to 4 , with the descriptions provided in the previous section). Women were given no other instruction on CMM, were not required to perform CMM, and were given no information about its potential utility for identifying the fertile window or optimizing intercourse timing. Participants were asked to complete the diary daily until their first positive pregnancy test or four months of completed charting if no pregnancy occurred. After the fourth month the women were asked to complete a similar diary only once per month for up to 12 months of enrollment or until pregnancy occurred. The women were given free home pregnancy tests (sensitivity: 20 mIU human chorionic gonadotropin $/ \mathrm{mL}$ ) and were instructed to use them at the time of a missed menses and inform the study staff of a positive result.

\section{Analysis}

The TTC study did not require the collection of systematic biomarker or symptom data across all women or all cycles in the study, so the fertile widow was estimated using calendar calculations based on cycle length $(5,17)$. Ovulation was estimated to have occurred 14 days before the first day of menses or the first positive home pregnancy test, with the fertile window designated as extending from 5 days before to 3 days after the estimated day of ovulation as defined previously. The use and frequency of CMM was determined for each cycle by the women's responses in the daily diary. The percentage of days of CMM from the first day after the cessation of menses through the end of the fertile window was calculated for each woman in each cycle. Given that CMM varied across cycles, each cycle for each woman was independently categorized as non-monitored (did not record a CM score on any of the days from the first day after menses through the fertile window), infrequently monitored (mucus checked on $1 \%$ to $33 \%$ of days), inconsistently monitored ( $34 \%$ to $66 \%$ of days), or consistently monitored (>66\% of days). Pregnancy was defined by the first report of a positive home pregnancy test.

Pearson correlations, Kruskal-Wallis tests, and chisquare tests were used to compare demographics and potential covariates (age, race, marital status, education level, smoking, previous pregnancy, body mass index [BMI], intercourse frequency in the fertile window, urinary LH monitoring, past hormonal contraception, partner age, partner race, partner BMI, and partner education) between the categories of CMM during the first completed cycle in the study.

The potential covariates were subsequently examined via bivariate analysis and likelihood ratio testing. For the models, we included the covariates that were strongly associated with fecundability in our study or that had been identified in multiple prior studies as related to fecundability, even if these variables were not statistically significant in our study. Potential covariates that were highly correlated with other predictors thought to have a greater relevance (i.e., partner age is highly correlated with subject age) were excluded. Unadjusted discrete-time survival models were subsequently created to assess the relationship between the covariates and fecundability.

These models treated an attempt cycle as the time unit of interest, using the "discrete" method to handle ties and account for differential attempt cycles at the time of study entry. 
Maternal age was collapsed into three categories for modeling (30-34, 35-37, and 38-44 years of age), partner age was dichotomized as $<50$ or $\geq 50$ years of age, and BMI was categorized into three groups $\left(<25,25.0-29.9\right.$, and $\left.230 \mathrm{~kg} / \mathrm{m}^{2}\right)$.

A discrete time proportional hazards model with time-varying (cycle-specific) categories of CMM, intercourse frequency, and urinary LH monitoring was created to analyze the effect of CMM in a given cycle (as recorded in the daily diary) on the probability of pregnancy in that cycle. This model accounts for both the right censoring and left truncation (due to women enrolling in cycles 1, 2, 3, or 4 of their pregnancy attempt) present in the data. In this model, a fecundability ratio (FR) less than 1.0 suggests reduced fecundability. The final model included maternal age, race (non-Hispanic white or non-white), history of previous pregnancy (dichotomized as any/none), maternal BMI (calculated using subject-reported height and weight), and cycle-specific intercourse frequency during the fertile window (percentage of days in the fertile window with intercourse). A model was created with an interaction term to assess for potential effect modification by urinary LH testing, but the interaction was found to be statistically nonsignificant and was excluded from the final model.

\section{Results}

A total of 448 women were enrolled in the study between April 2008 and November 2011. Seventy-four percent $(n=331)$ of the women completed the baseline survey and at least one cycle of daily diaries and were therefore included in this analysis. There were no statistically significant differences in the covariates examined in this study between the women who completed the daily diaries and those who did not. Of the analysis cohort, $70 \%$ of women were $<35$ years of age, $19 \%$ were $35-37$ years, and $11 \%$ were 38 years or older. Participants tended to be Caucasian (76\%), highly educated (71\% with a master's degree or $\mathrm{PhD}$ ), previously pregnant $(57 \%)$, and of normal BMI $(62 \%)$. Sixteen percent of participants were obese (BMI 230) and 2\% were underweight (BMI < 18.5). Twenty-three percent of the women enrolled became pregnant during their first cycle; by 6 months of enrollment, at least $53 \%$ of the women had conceived.

During the first cycle in the study, 178 (54\%) of the women did not use CMM, 73 (22\%) monitored infrequently, 60 (18\%) monitored inconsistently, and $20(6 \%)$ monitored consistently. The women who performed CMM (in the first cycle) tended to be younger (Pearson correlation of $-0.14, P=.010$ ) and were more likely to use urinary LH monitoring ( $P=.01$ ). Women performing consistent $\mathrm{CMM}$ were more likely to be nulligravid ( $P=.002)$. There were no differences in regards to race, BMI, education, smoking, intercourse frequency, or past hormonal contraceptive use (Table 1).

In subsequent cycles, $22 \%$ of the women increased the frequency of CMM, 37\% decreased, $38 \%$ remained the same, and 3\% both increased and decreased their frequency of CMM. After excluding the women who had conceived in the first cycle and adjusting for baseline CMM category, age, BMI, race, previous pregnancy, intercourse frequency, and LH use, increasing CMM category was not statistically significantly associated with fecundability ( $\mathrm{FR}=1.57,95 \% \mathrm{CI}, 0.75-3.31)$ where no change in CMM was the referent group. However, this analysis was likely underpowered (only 240 women) to determine the association between change in CMM category and fecundability. Across all cycles, CM was monitored (including infrequent, inconsistent, and consistent categories) in $42 \%$ of cycles. Of all CM observations, type 3 was the most commonly reported (40\%) and type 4 the least (15\%). Twenty-five percent of observations were documented as type $1 \mathrm{CM}$ and $20 \%$ as type 2 . 
The unadjusted and adjusted fecundability ratios for consistency of CMM are presented in Table 2. Cycles in which women consistently performed CMM were statistically significantly more likely to result in conception after adjusting for age, race, previous pregnancy, BMI, and LH monitoring ( $\mathrm{FR}=2.29$; 95\% confidence interval [CI], 1.22-4.32), and they demonstrated higher cumulative pregnancy rates (Fig. 1) compared with the cycles in which women did not perform CMM. The association between CMM and fecundability did not differ among women who did or did not perform urinary LH monitoring ( $\mathrm{P}_{\text {interaction }}$ $=0.9073$; data presented in Supplemental Table 1, available online), and the benefit of $\mathrm{CMM}$ remained after adjusting for intercourse frequency in the fertile window ( $\mathrm{FR}=2.32$; $95 \% \mathrm{CI}, 1.23-4.40)$. A trend of increasing fecundability with increasing frequency of CMM was also noted $(P=.01)$.

\section{Discussion}

In our study, we found that consistent CMM was an infrequently used method of detecting the fertile window in highly educated, noninfertile women, 30 to 44 years of age, who were trying to conceive. The women who performed CMM were on the younger side of our participants, and they tended to be more frequent users of urinary LH monitoring. CMM was associated with increased fecundability independent of the use of urinary LH monitoring and intercourse frequency. Furthermore, there was a statistically significant dose response, with the level of consistency of CMM being positively associated with increasing fecundability.

We found that any use of CMM ( $42 \%$ of participants) was more common than the use of urinary LH (27\%) or basal body temperature monitoring (30\%) in our study; however, consistent CMM was less common than the other methods. Our findings may be related to our relatively older reproductive cohort; we found that CMM prevalence decreased with increasing maternal age. Younger women who have not previously conceived may proactively seek resources to improve fertility. Younger women are more frequent users of the Internet in general, and are also more likely to participate in blogs, seek online support groups, and use online social networking $(18,19)$, which gives them easy access to information on CMM. Our cohort was highly educated, which has been implicated in frequency of overall Internet use, although a large national survey of over 7,000 participants found that age was the biggest predictor of blog, support group, and social networking use (19). We did not, however, assess whether the women in our older reproductive cohort had sought CMM resources. Although age was inversely associated with CMM, adjustment for age in our models did not diminish the impact of CMM on fecundability.

Compared with the women who did not monitor, CMM was associated with increased fecundability. Urinary LH monitoring combined with monitoring for urinary estrone glucuronide has also been associated with increased odds of conception (OR 1.89; 95\% CI, $1.24-2.89)$ and a shortened time to pregnancy $(20,21)$. The benefits of the combined monitoring based on the ClearBlue Easy Fertility Monitor (CBEFM) were statistically significant only during the early attempts to conceive (all less than 6 months) (20). Our study also included women early in their attempts to conceive, so we cannot comment on the benefits of CMM for women who are further along in their attempts. No trials have compared the utility of urinary LH monitoring with CMM using time to pregnancy as the outcome. A study comparing the CBEFM (formerly the ClearPlan Easy Fertility Monitor) with CMM demonstrated that both methods were capable of identifying the days of peak fertility; however, the CBEFM failed to identify the onset of the fertile window approximately $40 \%$ of the time (22). Although women who performed CMM in our study were more frequent users of urinary LH monitoring, the association between CMM and fecundability did not differ between those who did or did not perform urinary LH monitoring. 
Increasing consistency of CMM was also associated with increasing fecundability. Women who performed inconsistent CMM were less likely to conceive than the women performing consistent CMM. Thus, our findings support the recommendation that women monitor CM on a daily basis outside of menstruation to determine the fertile window, to time intercourse, and to maximize the probability of conception. Although one would presume that increasing frequency of urinary LH monitoring is also associated with increasing fecundability, to our knowledge this has not been assessed in any published studies.

Our study suggests that CMM reduces the time to pregnancy by improving the timing of intercourse. The number of acts of intercourse in the fertile window (as defined by calendar calculations) did not differ by use of CMM. This is in agreement with prior research findings that CMM provides additional information above and beyond that available through calendar calculations $(14,15)$, likely due to the fact that the timing of the fertile window varies substantially even in women with normal menstrual cycles (23). We propose that CMM is more effective for the timing of intercourse than the calendar method, as it allows for the onset and duration of the fertile window to be determined prospectively (22). It is also possible that the association between CMM and improved fecundability was due to residual confounding, even though we adjusted for likely confounders such as age, previous pregnancy, intercourse frequency, urinary LH monitoring, and BMI.

To our knowledge, ours is the first study to assess CMM use and efficacy in a noninfertile population without formal $\mathrm{CM}$ training. The strengths of our study include the use of a daily diary, which helped minimize recall bias. CMM was not included in the study literature or instructions, and it was reported prospectively before pregnancy. Also, the standardized protocol for pregnancy testing increased the likelihood and accuracy of outcome reporting. Our study does have limitations. Although the women were not instructed on how to perform CMM, the daily diary could be considered informative because it included a description of each CM type. Furthermore, our study cohort was well educated, lacked women of younger reproductive ages, and only included women early in their attempts to conceive. Additionally, although the effect of alcohol and caffeine on female fertility has not been clearly established, some studies have suggested detrimental effects on fertility, potentially in a dose-dependent manner $(24,25)$. Our analysis did not adjust for alcohol or caffeine consumption, potentially contributing to residual, unmeasured confounding. However, our positive findings support the need for a randomized controlled trial of CMM in women of all reproductive ages in the general population.

Our study demonstrates that although sporadic use of CMM is common, consistent CMM is infrequent in a population of women trying to conceive. However, even without formal training, consistent CMM was associated with increased fecundability independent of intercourse frequency or urinary LH monitoring. This suggests that CMM-a free, selfdirected method used to detect the fertile window—could potentially be used alone to increase fecundability. It is further possible that more widespread use of CMM could potentially lower the incidence of infertility and the need for fertility services in the general population.

\section{Supplementary Material}

Refer to Web version on PubMed Central for supplementary material.

\section{Acknowledgments}

Supported by National Institute of Child Health and Human Development grants R21-HD060229 and R01HD067683, and National Institute of Environmental Health Sciences grant T32ES007018. 


\section{References}

1. Wilcox AJ, Weinberg CR, Baird DD. Timing of sexual intercourse in relation to ovulation. Effects on the probability of conception, survival of the pregnancy, and sex of the baby. N Engl J Med. 1995; 333:1517-21. [PubMed: 7477165]

2. Dunson DB, Baird DD, Wilcox AJ, Weinberg CR. Day-specific probabilities of clinical pregnancy based on two studies with imperfect measures of ovulation. Hum Reprod. 1999; 14:1835-9. [PubMed: 10402400]

3. Chandra A, Martinez GM, Mosher WD, Abma JC, Jones J. Fertility, family planning, and reproductive health of U.S. women: data from the 2002 National Survey of Family Growth. Vital Health Stat. 2005; 23:1-160.

4. Stanford JB, White GL, Hatasaka H. Timing intercourse to achieve pregnancy: current evidence. Obstet Gynecol. 2002; 100:1333-41. [PubMed: 12468181]

5. Mikolajczyk RT, Stanford JB. Measuring fecundity with standardised estimates of expected pregnancies. Paediatr Perinat Epidemiol. 2006; 20(Suppl 1):43-50. [PubMed: 17061973]

6. McGovern PG, Myers ER, Silva S, Coutifaris C, Carson SA, Legro RS, et al. Absence of secretory endometrium after false-positive home urine luteinizing hormone testing. Fertil Steril. 2004; 82:1273-7. [PubMed: 15533341]

7. Miller PB, Soules MR. The usefulness of a urinary LH kit for ovulation prediction during menstrual cycles of normal women. Obstet Gynecol. 1996; 87:13-7. [PubMed: 8532248]

8. Hilgers TW, Bailey AJ, Prebil AM. Natural family planning IV. The identification of postovulatory infertility. Obstet Gynecol. 1981; 58:345-50. [PubMed: 7266954]

9. Quagliarello J, Arny M. Inaccuracy of basal body temperature charts in predicting urinary luteinizing hormone surges. Fertil Steril. 1986; 45:334-7. [PubMed: 3949032]

10. Luciano AA, Peluso J, Koch EI, Maier D, Kuslis S, Davison E. Temporal relationship and reliability of the clinical, hormonal, and ultrasonographic indices of ovulation in infertile women. Obstet Gynecol. 1990; 75:412-6. [PubMed: 2406661]

11. Bigelow JL, Dunson DB, Stanford JB, Ecochard R, Gnoth C, Colombo B. Mucus observations in the fertile window: a better predictor of conception than timing of intercourse. Hum Reprod. 2004; 19:889-92. [PubMed: 14990542]

12. Stanford JB, Smith KR, Dunson DB. Vulvar mucus observations and the probability of pregnancy. Obstet Gynecol. 2003; 101:1285-93. [PubMed: 12798538]

13. Dunson DB, Sinai I, Colombo B. The relationship between cervical secretions and the daily probabilities of pregnancy: effectiveness of the two day algorithm. Hum Reprod. 2001; 16:227882. [PubMed: 11679504]

14. Scarpa B, Dunson DB, Colombo B. Cervical mucus secretions on the day of intercourse: an accurate marker of highly fertile days. Eur J Obstet Gynecol Reprod Biol. 2006; 125:72-8. [PubMed: 16154254]

15. Scarpa B, Dunson DB, Giacchi E. Bayesian selection of optimal rules for timing intercourse to conceive by using calendar and mucus. Fertil Steri. 2007; 88:915-24.

16. Steiner AZ, Herring AH, Kesner JS, Meadows JW, Stanczyk FZ, Hoberman S, et al. Antimullerian hormone as a predictor of natural fecundability in women aged 30-42 years. Obstet Gynecol. 117:798-804. [PubMed: 21422850]

17. Colombo B, Scarpa B. Calendar methods of fertility regulation: a rule of thumb. Statistica. 1996; 56:3-14. [PubMed: 12348075]

18. Morales, L. Nearly Half of Americans are Frequent Internet Users. Washington DC: Gallup Poll; 2009.

19. Chou WY, Hunt YM, Beckjord EB, Moser RP, Hesse BW. Social media use in the United States: implications for health communication. J Med Internet Res. 2009; 11:e48. [PubMed: 19945947]

20. Robinson JE, Wakelin M, Ellis JE. Increased pregnancy rate with use of the Clearblue Easy Fertility Monitor. Fertil Steril. 2007; 87:329-34. [PubMed: 17074329]

21. Robinson JE, Ellis JE. Mistiming of intercourse as a primary cause of failure to conceive: results of a survey on use of a home-use fertility monitor. Current Medical Research and Opinion. 2007; 23:301-6. [PubMed: 17288684] 
22. Fehring RJ, Raviele K, Schneider M. A comparison of the fertile phase as determined by the Clearplan Easy Fertility Monitor and self-assessment of cervical mucus. Contraception. 2004; 69:9-14. [PubMed: 14720613]

23. Wilcox AJ, Dunson D, Baird DD. The timing of the "fertile window" in the menstrual cycle: day specific estimates from a prospective study. BMJ. 2000; 321:1259-62. [PubMed: 11082086]

24. Eggert J, Theobald H, Engfeldt P. Effects of alcohol consumption on female fertility during an 18year period. Fertil Steril. 2004; 81:379-83. [PubMed: 14967377]

25. Bolumar F, Olsen J, Rebagliato M, Bisanti L. Caffeine intake and delayed conception: a European multicenter study on infertility and subfecundity European Study Group on Infertility Subfecundity. Am J Epidemiol. 1997; 145:324-34. [PubMed: 9054236] 

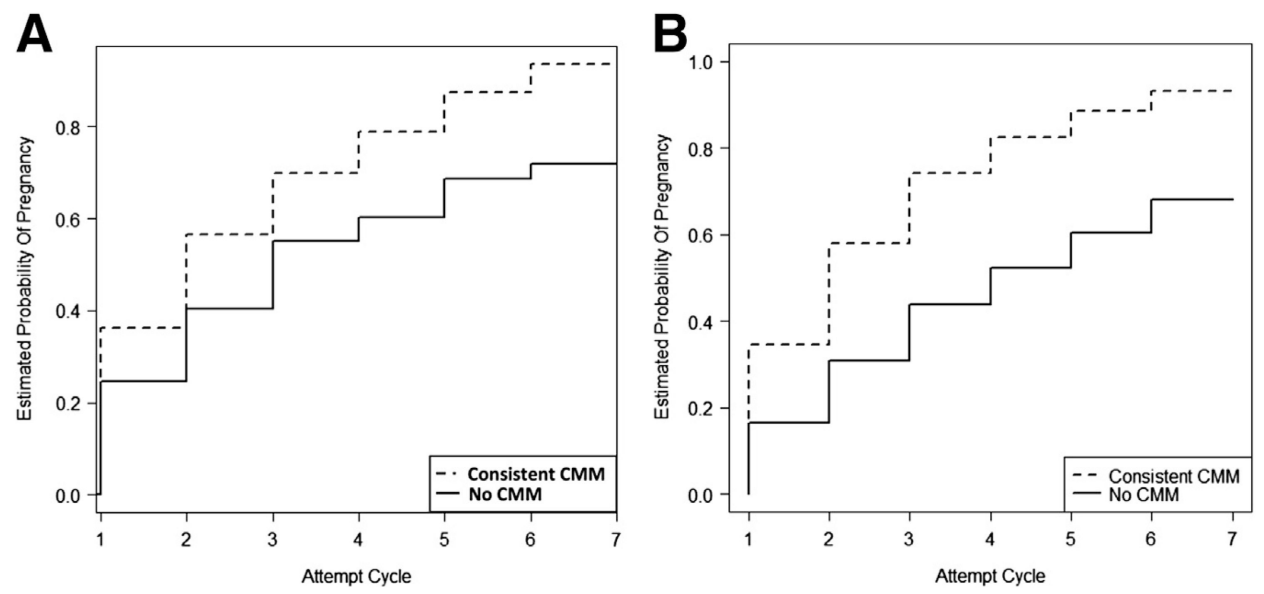

Figure 1.

(A) Unadjusted, Kaplan-Meier curves with time varying CMM. (B) Adjusted, discrete-time proportional hazards model using first cycle information on CMM, intercourse frequency during the fertile window, and LH monitoring $(P=.02)$. 
$\stackrel{\frac{\pi}{3}}{\frac{\pi}{2}}=$

ๆิ

ఠิ $\quad=\quad \stackrel{+}{\infty}$

:

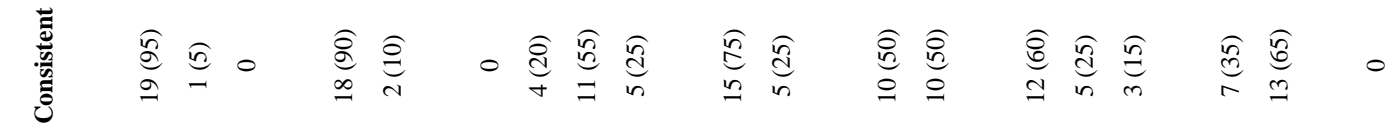

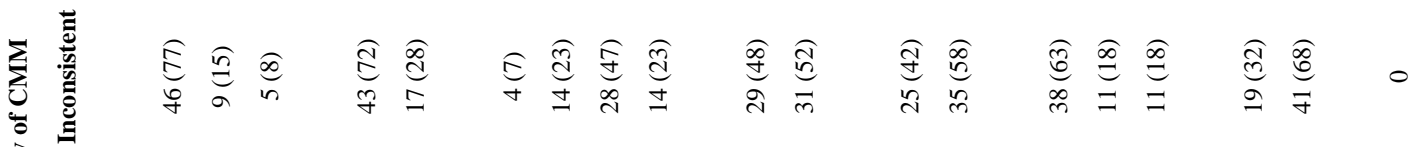

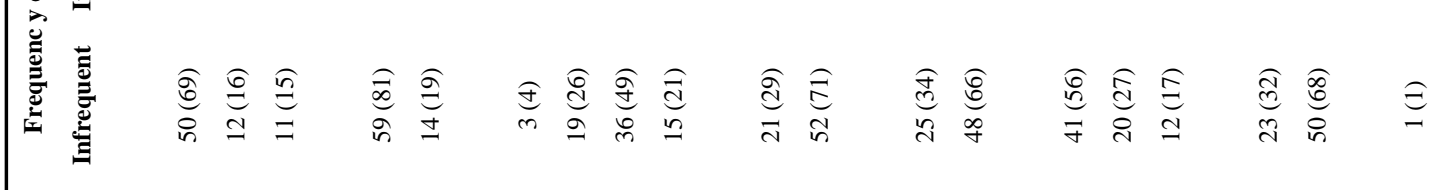

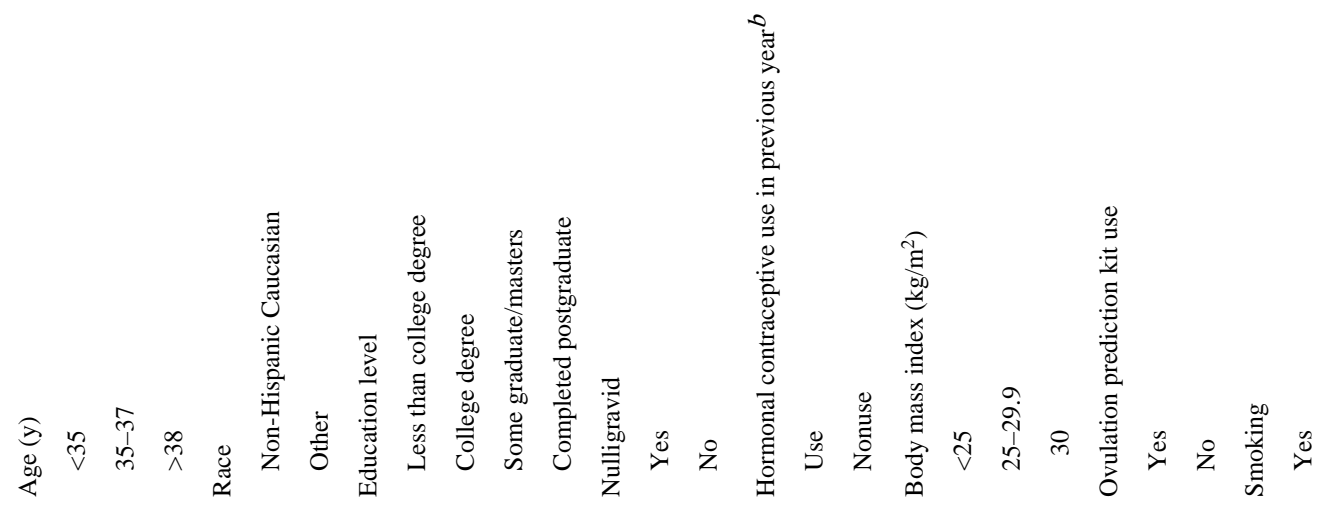




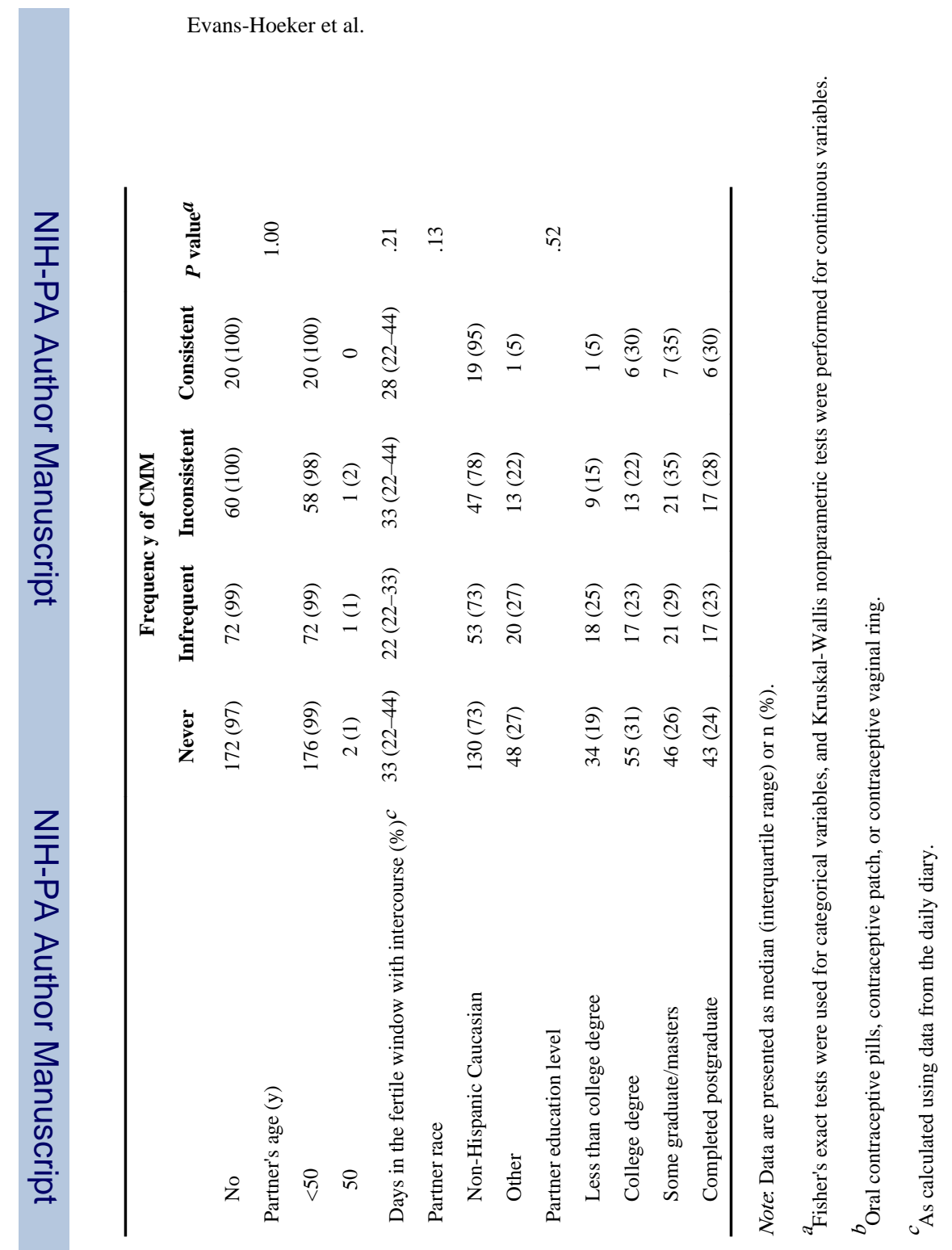

Fertil Steril. Author manuscript; available in PMC 2014 October 01. 


\section{Table 2}

Cycle-specific fecundability ratios (FR) with $95 \%$ confidence intervals (CI) by category of cervical mucus monitoring (CMM).

\begin{tabular}{lcccc}
\hline & \multicolumn{4}{c}{ Fecundability ratio } \\
& \multicolumn{2}{c}{ Unadjusted } & \multicolumn{3}{c}{ Adjusted $^{a}$} \\
Category of CMM & FR & $\mathbf{9 5 \%}$ CI & FR & 95\% CI \\
None & \multicolumn{4}{c}{ Reference } \\
Infrequent ( $33 \%)$ & 1.05 & $(0.68,1.62)$ & 1.10 & $(0.71,1.74)$ \\
Inconsistent $(34-66 \%)$ & 1.30 & $(0.79,2.16)$ & 1.36 & $(0.81,2.30)$ \\
Consistent $(>66 \%)$ & 2.10 & $(1.16,3.81)$ & 2.32 & $(1.23,4.40)$ \\
\hline
\end{tabular}

${ }^{a}$ Adjusted for age, race, previous pregnancy, BMI, urinary LH monitoring, and intercourse frequency. 